\section{$\S 25$. Acceleration Characteristics of Spherical and Non-Spherical Pellets by the LHD Impurity Pellet Injector}

Nozato, H., Takase, Y., Ejiri, A. (Frontier Sci., Univ. Tokyo)

Morita, S., Goto, M.

An impurity pellet injector ${ }^{1)}$ has been developed to study the particle/impurity transport in combination with a high-spatial resolution bremsstrahlung profile diagnostic ${ }^{2)}$ in the LHD plasma. The pellet injector consists of three differential pumping systems and three fast closing valves, as shown in Fig.1. A rotary pellet disk can load up to one hundred pellets at once and the pellet is accelerated by pressurized $\mathrm{He}$ gas. The maximum diameter of the available pellet is $1.0 \mathrm{~mm}$ and is limited by the inner diameter of the $1 \mathrm{~m}$ long acceleration pipe. The pellet velocity is measured at chambers M1, M2 and M3. After injecting the pellet, fast closing gate valves $\mathrm{V} 1, \mathrm{~V} 2$ and $\mathrm{V} 3$ are closed quickly to avoid He gas leakage into the LHD vacuum vessel. The vacuum pressure at chamber M3 is kept to the order of $10^{-9}$ torr. The time for closing the gate valves is approximately $5 \mathrm{~ms}$.

Measured pellet velocities at a $\mathrm{He}$ gas pressure of $18 \mathrm{~atm}$ and an acceleration valve opening time of $1.4 \mathrm{~ms}$ are summarized as a function of $A_{p} / M\left(m^{2} / \mathrm{kg}\right)$, where $A_{p}$ is the cross section and $M$ is the mass of the pellet (Fig.2). Linear relationships were found between the pellet velocity and $\mathrm{A}_{\mathrm{p}} / \mathrm{M}$ for spherical and cylindrical pellets. However, the data for semispherical pellets are somewhat scattered. It is probably due to the variation in the cross sectional area for the hemispherical pellet, in contrast to spherical and cylindrical pellets in which the cross section is always $\pi r^{2}$.

The pellet velocity $\mathrm{U}(\mathrm{t})$ and the barrel length $\mathrm{L}$ are given by

$$
U(t)=\frac{2 C_{0}}{\gamma-1}\left\{1-\left[1+\frac{(\gamma+1) A_{p}}{2 M C_{0}} P_{0} t\right]^{\frac{\gamma-1}{\gamma+1}}\right\}
$$

and

$L=\int U(t) d t=\frac{2 C_{0}}{\gamma-1}\left\{t-\frac{M C_{0}}{A_{p} C_{0}}\left[1+\frac{(\gamma+1) A_{p} P_{0}}{2 M C_{0}} t\right]^{\frac{2}{\gamma+1}}\right\}+\frac{2 M C_{0}{ }^{2}}{(\gamma-1) A_{p} P_{0}}$

,where $C_{0}$ is the propellant gas sound speed, $\gamma$ is the ratio of specific heats, and $\mathrm{P}_{0}$ is the gas pressure. By solving the equations simultaneously, the velocity for a cylindrical pellet can be obtained (Fig.2). All the velocities range from 10 and $40 \%$ of the theoretical values. Since the equation are developed for an ideal pellet, the gap between the pellet and the inner surface of the acceleration pipe is not taken into account. The existence of the gap gives rise to a reduction of the pellet velocity from the calculated value.

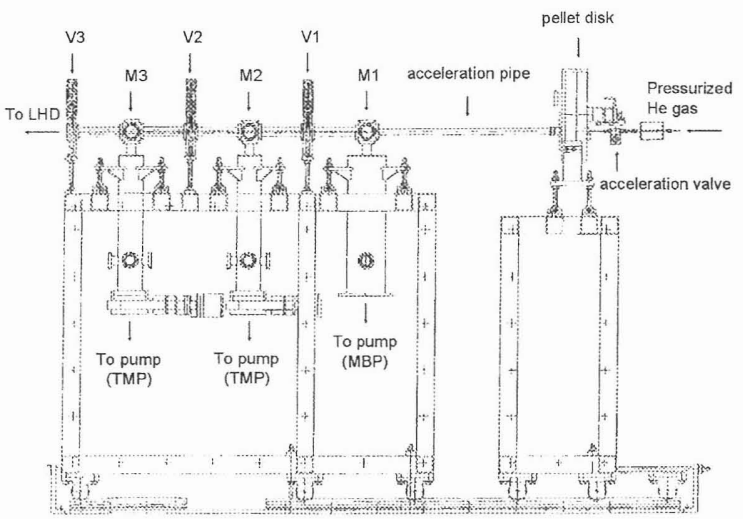

Fig.1. Side view of the LHD impurity pellet injector with 3 differential pumping systems and 3 fast closing valves $(\mathrm{V} 1, \mathrm{~V} 2, \mathrm{~V} 3)$. Vacuum chambers indicated by M1, M2 and M3 are used to measure the pellet velocity.

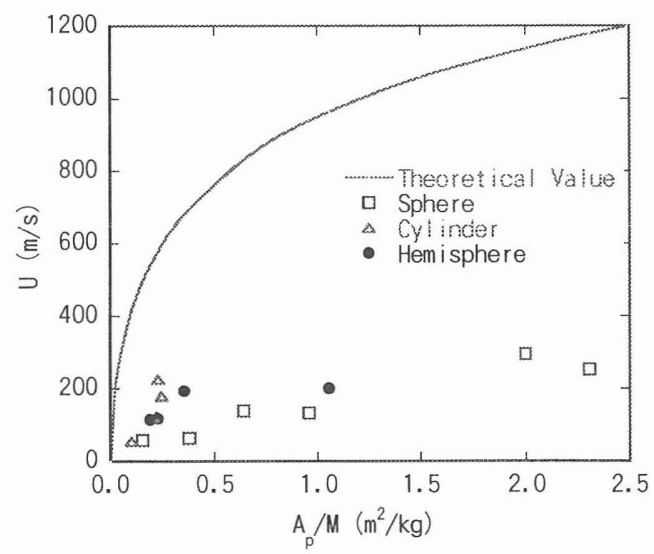

Fig. 2. Dependencies of pellet velocities of various shapes on $A_{p} / M\left(\mathrm{~m}^{2} / \mathrm{kg}\right)$ and a theoretical curve for a cylindrical pellet

\section{References}

1) Nozato, H., et al., Rev. Sci. Instrum. 74 (2003) 2032.

2) Nozato, H., et al., J. Plasma Fusion Res. SERIES 5 (2002) 442 . 